\title{
Research on Routing Choice of Computer Network Based on Improved Quantum Evolutionary Algorithm
}

\author{
Xin $\mathrm{Cao}^{\mathrm{a}}$, Yana Zhang ${ }^{\mathrm{b}}$ \\ Department of Software Engineering, Shijiazhuang Information Engineering Vocational \\ College,Shijiazhuang, 050035, China \\ a382173043@qq.com, bynzym105@sina.com
}

\begin{abstract}
Keywords: Improved quantum evolutionary algorithm; Rotation angle optimization; Routing; Computer network
\end{abstract}

Abstract. According to the problem of poor optimization performance and convergence performance of the current computer network's optimal routing algorithm, this paper puts forward the improved quantum evolutionary algorithm based on computer network routing. On the basis of traditional quantum evolutionary algorithm, this algorithm adjusts and optimizes the rotation Angle, speeds up the search speed, and improves the accuracy of optimization, also according to the quadrant of the qubits's space and phase Angle, this algorithm adjusts and optimizes the direction of rotation angle. Algorithm simulation results show that the proposed improved quantum evolutionary algorithm based on computer network routing shows a better optimization and convergence ability in computer network's routing choice than the traditional quantum evolutionary algorithm.

\section{Introduction}

Computer network is widely used in various social fields. Under the condition of a known network topology premise of each node needs to communicate, the main problem in planning or expansion of computer networks is how to choose the best computer communication network links route[1,2]. But this problem is with a lot of constraints and complex non-linear programming, belonging to a class of NP-complete problem in combinatorial optimization. The traditional mathematical theory has no effective solution method. The main purpose of this paper is improve the traditional quantum evolutionary algorithm through the selection of mathematical models by computer network routing.

\section{The mathematical model of routing}

The selection problem of communication network routing can be summarized as: in the premise of the known network topology, link capacity and the requirement of each node to communication, determine the communication route for each node, so that the average delay of the network is minimum. Based on queuing theory, construct a $M / M / 1$ queuing model, one of link $l$ is packet, and obtain the average of the extended time:

$$
T_{l}=\frac{1}{\mu Q_{l}-\lambda_{l}}
$$

Where the time extend value of link packet is defined as $T_{l} ; Q_{l}$ represents the capacity of $l$-th communication links; $\lambda_{l}$ represents the packet arrival rate of the $l$-th communication link; $1 / \mu$ 
represents the average length of link packet, namely the average of the probability density $\mu e^{-\mu t}$.

Weight all links in the length of time, then the whole average time extend value of the network is expressed as follows:

$$
T=\frac{1}{\lambda} \sum_{l \in L} \frac{\sum_{r \in R} \lambda_{r} \delta_{r l} x_{r}}{\mu Q_{l}-\sum_{r \in R} \lambda_{r} \delta_{r l} x_{r}}
$$

Thus, the selection optimal state of the communication network routers can be described by mathematical models. The concrete description is shown as follows:

$$
\min \left(T=\frac{1}{\lambda} \sum_{l \in L} \frac{\sum_{r \in R} \lambda_{r} \delta_{r l} x_{r}}{\mu Q_{l}-\sum_{r \in R} \lambda_{r} \delta_{r l} x_{r}}\right)
$$

In this state, its constraints are as follows:

$$
\begin{aligned}
& Q_{l} \geq \frac{1}{\mu} \sum_{r \in R} \lambda_{r} \delta_{r l} x_{r} \quad(\forall l \in L) \\
& \sum_{r \in S_{p}} x_{r}=1 \quad(\forall p \in \Pi) \\
& x_{r}=0,1 \quad(\forall r \in R)
\end{aligned}
$$

Where $\Pi$ represents the set of all communication node pairs in the network; $L$ represents the set of all the links in the network; $R$ represents the candidate route set; $\delta_{r l}$ represents the identification of the function, when the route $r$ contains link $l$, the value is 1 , otherwise, the value is $0 . S_{p}$ represents the candidate routing set for nodes to $p(p \in \Pi)$, and set $S_{p} \cap S_{q}$ as an empty set. $\lambda_{r}$ represents the data packets arrival rate related to routing $r ; x_{r}$ represents the optimization variable, when the communication routing is related to the node, its value is 1 , otherwise, the value is $0 ; \lambda$ represents total network packet arrival rate, and $\lambda=\sum_{p \in \Pi} \lambda_{p}$.

\section{The quantum evolution algorithm}

In quantum evolutionary algorithm, the following matrix can be used to express quantum chromosome, and its length is $m$.

$$
\left[\begin{array}{ccccc}
\alpha_{1} & \cdots & \alpha_{i} & \cdots & \alpha_{m} \\
\beta_{1} & \cdots & \beta_{i} & \cdots & \beta_{m}
\end{array}\right]
$$

Where $\alpha_{i}$ represents the probability amplitude of qubit states $|0\rangle ; \beta_{i}$ represents the probability amplitude of qubit states $|1\rangle$, and it needs to satisfy the following normalization condition: 


$$
\left|\alpha_{i}\right|^{2}+\left|\beta_{i}\right|^{2}=1 \quad(i=1,2, \cdots, m)
$$

The superposition state of all solutions in solution space can be characterized by a quantum chromosome, mainly through random measurement results of quantum chromosomes. The achievement of collapse solution is completed by binary according to the probability. And it can be seen that the quantum chromosome is the best reflection for keeping the diversity of solutions. Thus, it need quantum revolving door to complete the evolutionary strategy of quantum plan algorithm[3].

When the algorithm updates to the $t$-th iteration $P(t)=\left\{p_{1}^{t}, \cdots, p_{N}^{t}\right\}$, its fitness value is defined as $f(t)=\left\{f_{1}^{t}, \cdots, f_{N}^{t}\right\}$. The $i$-th value of the $p_{j}^{t}$ gene is expressed as $\left(\alpha_{i}, \beta_{i}\right)^{T}$, and make a quantum revolving door evolution for it and obtain $\left(\alpha_{i}^{\prime}, \beta_{i}^{\prime}\right)^{T}$. The concrete description is as follows:

$$
\left[\begin{array}{c}
\alpha_{i}^{\prime} \\
\beta_{i}^{\prime}
\end{array}\right]=\left[\begin{array}{cc}
\cos \theta_{i} & -\sin \theta_{i} \\
\sin \theta_{i} & \cos \theta_{i}
\end{array}\right]\left[\begin{array}{c}
\alpha_{i} \\
\beta_{i}
\end{array}\right]
$$

Where $\theta_{i}$ represents the rotation angle which is calculated by:

$$
\theta_{i}=\Delta \theta_{i} s\left(\alpha_{i}, \beta_{i}\right) \pi
$$

Where the value of $\Delta \theta_{i}$ and $s\left(\alpha_{i}, \beta_{i}\right)$ are obtained by Table. 1. $\Delta \theta_{i}$ represents quantum revolving door rotation angle value, which is a control for the convergence speed control. The direction control of quantum revolving door rotation angle is achieved by $s\left(\alpha_{i}, \beta_{i}\right)$, which can

\begin{tabular}{|c|c|c|c|c|c|c|c|}
\hline \multirow{2}{*}{$x_{i}$} & \multirow{2}{*}{$b_{i}$} & \multirow{2}{*}{$f_{x} \geq f_{b}$} & \multicolumn{4}{|c|}{$s\left(\alpha_{i}, \beta_{i}\right)$} & \multirow{2}{*}{$\Delta \theta_{i}$} \\
\hline & & & $\alpha_{i} \beta_{i}>0$ & $\alpha_{i} \beta_{i}<0$ & $\alpha_{i}=0$ & $\beta_{i}=0$ & \\
\hline 0 & 0 & $\mathrm{~F}$ & 0 & 0 & 0 & 0 & 0 \\
\hline 0 & 0 & $\mathrm{~T}$ & 0 & 0 & 0 & 0 & 0 \\
\hline 0 & 1 & $F$ & 0 & 0 & 0 & 0 & 0 \\
\hline 0 & 1 & $\mathrm{~T}$ & +1 & -1 & 0 & 0 & 0.05 \\
\hline 1 & 0 & $\mathrm{~F}$ & +1 & -1 & 0 & \pm 1 & 0.01 \\
\hline 1 & 0 & $\mathrm{~T}$ & -1 & +1 & \pm 1 & \pm 1 & 0.025 \\
\hline 1 & 1 & $F$ & -1 & +1 & \pm 1 & 0 & 0.005 \\
\hline 1 & 1 & $\mathrm{~T}$ & -1 & +1 & \pm 1 & 0 & 0.025 \\
\hline
\end{tabular}
ensure optimal algorithm to carry out the search.

Table. 1 Function lookup table

Since the quantum chromosome may occur the collapse of the binary solutions, resulting in the same solution, so the $s\left(\alpha_{i}^{\prime}, \beta_{i}^{\prime}\right)^{T}$ in Eq. (9) is processed as follows: 


$$
\left[\begin{array}{c}
\alpha_{i}^{\prime} \\
\beta_{i}^{\prime}
\end{array}\right]=\left\{\begin{array}{l}
(\sqrt{\varepsilon}, \sqrt{1-\varepsilon})^{T},\left|\alpha_{i}\right|^{2} \leq \varepsilon \\
(\sqrt{1-\varepsilon}, \sqrt{\varepsilon})^{T},\left|\alpha_{i}\right|^{2} \geq 1-\varepsilon \\
\left(\alpha_{i}^{\prime}, \beta_{i}^{\prime}\right)^{T}, \text { else }
\end{array}\right.
$$

Where $\varepsilon$ is a positive number, and its value is small.

\section{The improvement strategy}

In the process of population updating in quantum evolutionary algorithm, the adjustment strategy of $\Delta \theta_{i}$ and $s\left(\alpha_{i}, \beta_{i}\right)$ is the key. In this paper, the dynamic adjustment strategy is applied to improve them.

\section{The adjustment optimization of rotation angle.}

The adjustments of improved $\Delta \theta_{i}$ is expressed as follows:

$$
\Delta \theta_{i}=0.001 \pi \cdot 50 \frac{f_{b}-f_{x}}{f_{x}}
$$

Where $f_{b}$ represents the fitness of the best individual; $f_{x}$ represents the fitness of current individual. It can be analyzed from Eq. (12) that $\Delta \theta_{i}$ represents a range of values, and it adapts to the individual. The larger the value of $\Delta \theta_{i}$, the longer distance between the current individual and the optimal individual, and represent that the search network is larger, so it is necessary to improve search speed.

\section{The adjustment optimization of function.}

This paper presents a rotation angle adjustment strategy according to the size quadrant and phase angle of the quantum in a two-dimensional real space, which is shown in Table. 2.

\begin{tabular}{|c|c|c|c|c|}
\hline$\alpha_{i}^{b}$ & $\beta_{i}^{b}$ & $\alpha_{i}^{x}$ & $\beta_{i}^{x}$ & $s\left(\alpha_{i}^{x}, \beta_{i}^{x}\right)$ \\
\hline$>0$ & $>0$ & $>0$ & $>0$ & $\begin{array}{l}-1,\left(\left|\omega_{i}^{b}\right|>\left|\omega_{i}^{x}\right|\right) \\
+1,\left(\left|\omega_{i}^{b}\right|<\left|\omega_{i}^{x}\right|\right)\end{array}$ \\
\hline$>0$ & $>0$ & $<0$ & $>0$ & +1 \\
\hline$>0$ & $>0$ & $<0$ & $<0$ & $\begin{array}{l}+1,\left(\left|\omega_{i}^{b}\right|>\left|\omega_{i}^{x}\right|\right) \\
-1,\left(\left|\omega_{i}^{b}\right|<\left|\omega_{i}^{x}\right|\right)\end{array}$ \\
\hline$>0$ & $>0$ & $>0$ & $<0$ & -1 \\
\hline
\end{tabular}

Table. 2 Optimization strategy

In Table. 2, $\alpha_{i}^{b}$ and $\beta_{i}^{b}$ represent the probability amplitude of the $i$-th qubit on the optimal individual, and its phase angle is defined as $\omega_{i}^{b}=\operatorname{arctg}\left(\beta_{i}^{b} / \alpha_{i}^{b}\right)$. The value of probability 
amplitude to be updated of $i$-th qubit is expressed as $\alpha_{i}^{x}$ and $\beta_{i}^{x}$, and its phase angle is defined as $\omega_{i}^{x}=\operatorname{arctg}\left(\beta_{i}^{x} / \alpha_{i}^{x}\right) . s\left(\alpha_{i}^{x}, \beta_{i}^{x}\right)$ represents the rotation direction of qubit to be updated: Clockwise direction is represented by +1 , and -1 means counterclockwise. This rotation strategy can make current solution approach the the optimal solution, and accelerate the convergence speed.

\section{Simulation test}

In order to verify the performance of the proposed algorithm applying in routing selection of computer network. The simulation is implemented which is compared with the traditional quantum evolutionary algorithm. The simulation results are shown in Fig. 1 and Table. 3.

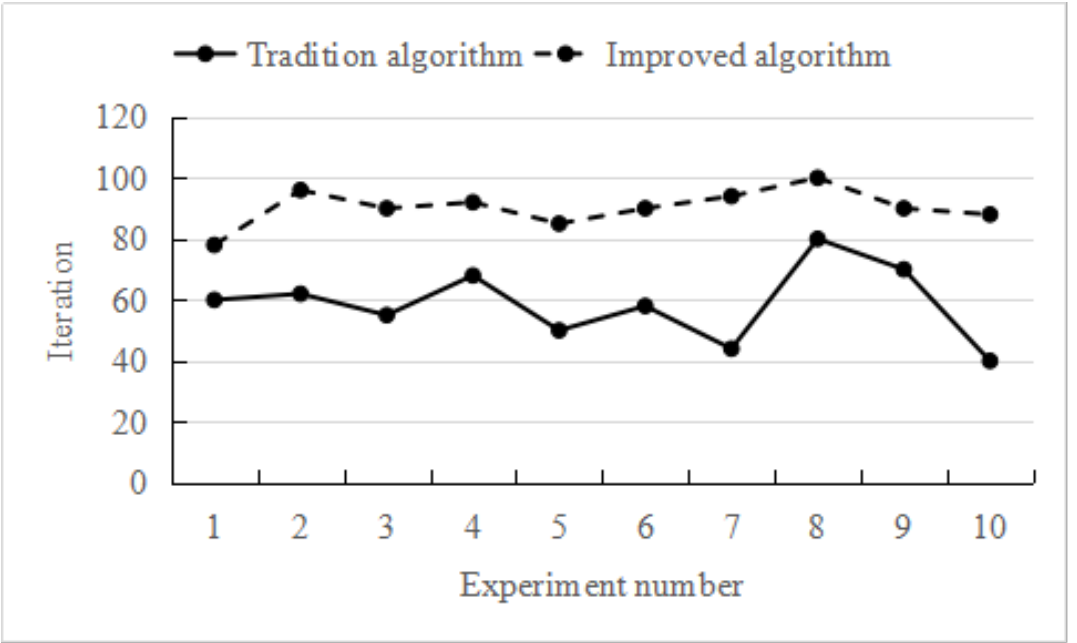

Fig. 1 Algorithm performance comparison

Table. 3 Routing simulation

\begin{tabular}{|c|c|c|c|c|}
\hline \multirow[b]{2}{*}{$\begin{array}{c}\text { Packet length } \\
\text { [bit] }\end{array}$} & \multicolumn{2}{|c|}{ Traditional algorithm } & \multicolumn{2}{|c|}{ Improved algorithm } \\
\hline & $\begin{array}{c}\text { The maximum of } \\
\text { link utilization } \\
{[\%]}\end{array}$ & $\begin{array}{c}\text { The average of } \\
\text { link utilization } \\
{[\%]}\end{array}$ & $\begin{array}{c}\text { The maximum of } \\
\text { link utilization } \\
{[\%]}\end{array}$ & $\begin{array}{c}\text { The average of } \\
\text { link utilization } \\
{[\%]}\end{array}$ \\
\hline 100 & 53.2 & 23.4 & 59.3 & 35.2 \\
\hline 110 & 55.3 & 25.3 & 63.4 & 37.1 \\
\hline 120 & 57.1 & 27.7 & 66.2 & 39.2 \\
\hline 130 & 59.3 & 29.3 & 67.1 & 42.6 \\
\hline 140 & 61.2 & 31.1 & 69.6 & 44.7 \\
\hline 150 & 63.4 & 33.2 & 71.3 & 48.3 \\
\hline
\end{tabular}

As can be seen from Fig. 1 and Table. 3, the proposed algorithm on optimization ability and convergence speed are superior to the traditional algorithm. Moreover, in the application of computer network routing selection, the performance of the proposed method is also better than that of the traditional algorithm.

\section{Conclusions}

This paper presented a computer network routing selection method based on improved quantum evolutionary algorithm. Through simulation experiments, based on the computer network routers, the improved quantum evolutionary algorithm is better than the traditional one on the convergence 
speed and optimization capability.

\section{References}

[1] Zhao Qingyan, Xiong Maohua. An improved Tabu search algorithm for wireless sensor network routing. Computer Measurement and Control, Volume 20, Issue 5 (2012), P. 1442-1444.

[2] Ding Wen. Multicast routing selection based on multi-object immune algorithm. Application Research of Computers, Volume 29, Issue 4, P. 1477-1479.

[3] Song Qianglei, Che Ada. Survey on application of quantum evolutionary algorithm in production scheduling. Application Research of Computers, Volume 29, Issue 5, P. 1601-1605. 